\title{
A NEW EFFICIENT NONLINEAR PROGRAMMING BASED METHOD FOR BRANCH OVERLOAD ELIMINATION
}

\author{
Heder D. Abrantes* \\ heder.abrantes@edb.ericsson.se \\ Carlos A. Castro* \\ ccastro@ieee.org \\ ${ }^{*}$ DSEE/FEEC/UNICAMP, P.O. Box 6101, 13081-970 Campinas SP, Brazil
}

\begin{abstract}
A simple and efficient method for eliminating branch overloads in power systems is presented in this paper. The overloads are eliminated by corrective control actions, which are defined through the use of an efficient and accurate nonlinear programming method. Generation rescheduling (GR) and load shedding (LS) are the main controls used. The idea of adaptative local optimization (ALO) is introduced. The computation of appropriate GR using ALO becomes a very efficient process. LS is used as a last resort, when further GR is no longer possible. Heuristics are added in order to speed up the computation process and to take into account some practical aspects of power systems operation into it. A special procedure is carried out in case of critical situations, where emergency control actions are defined. The method's general idea is to keep the new secure operating point as close as possible to the original one, while minimizing the amount of LS. The method can be a helpful tool for operation planning studies, security analysis and reliability evaluation of power systems. Simulations have been carried out for small test to large real life systems in order to show the efectiveness of the proposed method.
\end{abstract}

KEYWORDS: Power system security analysis, power system operation, violations elimination, nonlinear programming.

\footnotetext{
Artigo submetido em 30/11/00

1a. Revisão em 22/11/01; 2a. revisão em 25/04/02

Aceito sob recomendação do Ed. Assoc. Prof. José Luiz R. Pereira
}

\section{RESUMO}

Neste artigo é proposto um método simples e eficiente para eliminar sobrecargas em ramos de redes elétricas de potência. Essas sobrecargas são eliminadas através de ações de controle corretivo que são definidas através do uso de um método preciso e eficiente de programação não-linear. Redespacho da geração e corte de carga são os principais controle utilizados. A idéia de otimização local adaptativa é introduzida. O cálculo dos redespachos de geração utilizando a otimização local adaptativa é realizado de maneira muito eficiente. $\mathrm{O}$ corte de carga é utilizado como última alternativa, nas situações em que o redespacho da geração não é mais possível. Heurísticas são acrescentadas a fim de tornar o procedimento computacional mais rápido e de levar em conta alguns aspectos práticos da operação de redes elétricas de potência. Um procedimento especial é executado no caso de situações críticas, em que ações de controle de emergência são realizadas. A idéia geral do método é fazer com que o novo ponto de operação segura esteja o mais próximo possível de ponto de operação original, além de minimizar o montante de corte de carga. O método proposto pode ser uma ferramenta útil para estudos de planejamento, análise de segurança e avaliação da confiabilidade de sistemas de potência. Foram realizadas simulações para várias redes, incluíndo redes teste de pequeno porte e redes de grande porte reais, a fim de mostrar a eficácia do método proposto.

PALAVRAS-CHAVE: Análise de segurança de sistemas de potência, operação de sistemas de potência, eliminação de violações, programação não-linear. 


\section{INTRODUCTION}

Power systems operating conditions undergo frequent changes due to the occurence of outages and demand variations. These changes may result in one or more branch (transmission lines or transformers) MW overloads. In order to keep the system within secure operating conditions, corrective control actions must be taken so as to eliminate such overloads. Among the possible corrective control actions are generation rescheduling, interchange adjustments, phase shifters adjustments, network switching and load shedding (Wood and Wollenberg, 1984). Corrective control actions to eliminate overloads must be determined within a small time frame, specially in case of a real time control environment. They can also be determined by off-line calculations in order to provide a control strategy to be used in case of emergencies.

Several overload elimination methods have been proposed in the literature. In (Wood and Wollenberg, 1984; Castro and Bose, 1993; Castro and Bose, 1994) generation rescheduling methods based on generation shift factors were proposed. Heuristics were added in order to determine the controls to be used and to take into account some practical aspects of power systems operation. In (Medicherla et alii, 1979) bus injection changes were defined so as to decrease branch currents based on sensitivity factors. Nonlinear programming methods have also been proposed for finding coordinated control actions to eliminate overloads (Shandilya et alii, 1993). Optimization techniques are usually expensive from a computational standpoint. This cost increases with the size of the system. In (Shandilya et alii, 1993) the idea of local optimization (LO) is introduced. A fixed sized region (hereafter called work region) around the overloaded branch (or branches) is previously defined and corrective control actions are chosen within this region. Thus, the size of the problem to be solved is practically independent of the system size. Also, a scheme is proposed in an attempt to obtain minimum load shedding with maximum efficiency.

In this paper a nonlinear programming based method is proposed. Generation rescheduling and load shedding will be used as controls. The idea of LO (Shandilya et alii, 1993) has been extended to adaptive local optimization (ALO). Once the work region has been defined in LO, available generators for rescheduling may not be found. In ALO, the work region is defined so that generators with large sensitivities with respect to eliminating the overloads are always included. In (Shandilya et alii, 1993) all buses outside the work region are considered as slack buses (their states keep unchanged after control actions are taken into effect). Of course this is an approximation, since their states actually change after control actions are taken. In ALO, sparse vector techniques (Tinney et alii, 1985) are used and exact results are obtained with minimum computational effort. In addition, heuristics are added for increasing the overall efficiency of the computation process and coping with some practical aspects of the problem. Finally, emergency control measures are also allowed for critical overload cases for which usual generation rescheduling and load shedding are not sufficient. This provides the operator with a feasible solution for the problem, even though the system may have been driven farther away from its original operating point.

\section{PROBLEM FORMULATION}

In order to alleviate the existing overloads, a nonlinear optimization problem is formulated so as to minimize a quadratic function which includes the differences between the current MW power flows and their respective limits. This function will have as many terms as the number of overloaded branches. Let OL be the set of overloaded branches. Thus, the objective function is:

$$
f(\mathbf{x})=\sum_{\ell \in \mathrm{OL}}\left(P_{\ell}-P_{\ell}^{\max } \cdot \mathrm{sf}\right)^{2}
$$

where sf is a safety factor and $\mathbf{x}$ is the state variable vector (voltage magnitudes and phase angles). Function $f(\mathbf{x})$ can be minimized by changing bus MW power injections (generator rescheduling and load shedding). They are regarded as control variables. The constraints for this problem are:

$$
\begin{aligned}
& \mathbf{g}(\mathbf{x}, \mathbf{u})=\mathbf{0} \\
& \mathbf{h}(\mathbf{x}, \mathbf{u}) \leq \mathbf{0} \\
& \mathbf{u}^{\text {min }} \leq \mathbf{u} \leq \mathbf{u}^{\max }
\end{aligned}
$$

where $\mathbf{u}$ is the set of control variables.

Eq. (2) corresponds to the set of load flow equations. For practical purposes, MW power flows through branches depend basically on their phase angle spreads. Thus, equations that relate real powers and phase angles will are considered only. More specifically, the real power balance equation of the fast decoupled load flow formulation (Stott and Alsaç, 1974) is used. Cases for which power flows are affected by voltages magnitudes are appropriately taken care of in the solution process, as it will become clear in Sec. 3 .

Eq. (3) corresponds to branch MW power flow and bus voltage magnitude constraints. As far as branch MW power flows are concerned, most constraints are 
not violated at the solution point (most branches are not overloaded). Besides, the objective function specifically aims to satisfying the active constraints. As for bus voltage magnitudes, their limits may be considered in an appropriate manner in the solution process (Sec. $3)$. Therefore, (3) need not to be included in the basic formulation.

Eq. (4) takes into account the control variable lower and upper limits. In this case, only real power bus injections (generator rescheduling and load shedding) were considered. Finally, the problem is formulated as:

$$
\begin{aligned}
& f(\boldsymbol{\theta})=\sum_{\ell \in \mathrm{OL}}\left(P_{\ell}-P_{\ell}^{\mathrm{max}} \cdot \mathrm{sf}\right)^{2} \\
& \text { subject to } \\
& \mathbf{g}\left(\boldsymbol{\theta}, \mathbf{P}^{\mathrm{s}}\right)=\mathbf{P}^{\mathrm{s}}-\mathbf{P}^{\mathrm{c}}(\boldsymbol{\theta})=\mathbf{0} \\
& \mathbf{P}^{\text {min }} \leq \mathbf{P}^{\mathrm{s}} \leq \mathbf{P}^{\text {max }}
\end{aligned}
$$

where $\boldsymbol{\theta}$ is the nodal phase angle vector, and $\mathbf{P}^{\mathrm{s}}$ and $\mathbf{P}^{\mathrm{c}}$ are respectively the scheduled and calculated nodal real power injections. The elements of vector $\mathbf{P}^{\mathrm{c}}$ are computed by:

$$
P_{i}^{\mathrm{c}}=V_{i} \sum_{j \in A_{i}} V_{j}\left(G_{i j} \cos \theta_{i j}+B_{i j} \sin \theta_{i j}\right)
$$

where $V_{i}$ is the voltage magnitude at bus $i, G_{i j}$ and $B_{i j}$ are elements of the admittance matrix, and $A_{i}$ is a set containing bus $i$ plus the buses directly connected to it.

In this paper equations (5)-(7) are solved by a projected steepest descent method (Bazaraa and Shetty, 1993) where $\mathbf{P}^{\mathrm{s}}$ is the vector of independent variables and $\boldsymbol{\theta}$ is the vector of the dependent variables. See Appendix A for further details.

As a consequence of the utilization of $\mathrm{LO}$, the set of constraints (6) is formed by a small number of load flow equations (depending on the size of the work region). When ALO is used this set is formed by the load flow equations for all buses. However, they are solved as efficiently as in LO with the use of sparse vector techniques, with greater accuracy, as mentioned before.

The solution of the problem is obtained through a step by step procedure, each of which having a different control strategy. The control strategies defined are the following:

(1) generation rescheduling only;

(2) load shedding at the receiving end bus of the overloaded branches;
(3) load shedding at the buses to which power is being fed from the receiving end bus of the overloaded branches (first tier);

(4) change power injection (either rescheduling or load shedding) at buses located up to the second tier;

(5) change power injection (either rescheduling or load shedding) at buses located up to the third tier; and so on.

During the iterative process a specific control strategy is selected depending on the system condition at that iteration. The first three strategies have been proposed by (Shandilya et alii, 1993). The other ones are performed in case of persisting overloads and are referred to as emergency control measures. They assure that a feasible solution will be found in case of severe overloads. The determination of emergency control measures is very important to operators, specially when dealing with severe and hard to solve overload problems.

\section{PROPOSED ALGORITHM}

The complete procedure for alleviating branch overloads is as follows:

1 Perform load flow calculations using the scheduled load and generation information. Set strategy pointer $s p=1$ and outer iteration counter $o c=1$;

2 Calculate branch flows. If no overloads are found, go to step 12. If $s p>s p^{\max }$, stop. Else set inner iteration counter $i c=1$;

3 Calculate the value of the objective function $f_{0}$ at the current operating point;

4 If $s p>1$, determine the buses to be processed according to the current strategy, compute the respective elements of $\boldsymbol{\lambda}$ using Eq. (12) and go to step 6;

5 If $s p=1$, determine the work region using ALO. Starting from the terminal buses of the overloaded branches, expand the work region tier by tier in order to find generators for which their rescheduling would effectively alleviate the overloads. A generator is considered as an effective control if the sensitivity of the overloaded branch power flow with respect to a change in its power is above a certain threshold. This threshold is system dependent. The values of the lagrangean multipliers computed by Eq. (12) provide essentially the same information as sensitivity factors and were used in this paper. The search is interrupted in case generators 
with small sensitivities are found in two consecutive tiers. For each generator that is found, the corresponding value of vector $\boldsymbol{\lambda}$ is computed. In case the generator is not included as a control, the respective position is zeroed;

6 Calculate the conjugate of the direction given by $\boldsymbol{\lambda}$, that is, $\mathbf{S}=-\boldsymbol{\lambda}$;

7 Take into account the limits of the control variables, computing the projection of the gradients onto the bounds of the independent variables. The elements of vector $\mathbf{S}$ are redefined as:

$$
s_{j}=\left\{\begin{array}{l}
0 \quad \begin{array}{l}
\text { if } P_{j}^{\max }-P_{j}=0 \text { and } s_{j}>0 \\
\text { if } P_{j}-P_{j}^{\min }=0 \text { and } s_{j}<0
\end{array} \\
s_{j} \text { otherwise }
\end{array}\right.
$$

8 Calculate the maximum step size $t^{\mathrm{m}}$ such that:

$$
t^{\mathrm{m}}=\min _{j}\left\{\min _{s_{j}>0}\left[\frac{P_{j}^{\max }-P_{j}}{s_{j}}\right], \min _{s_{j}<0}\left[\frac{P_{j}^{\min }-P_{j}}{s_{j}}\right]\right\}
$$

9 Compute the optimum step size $t^{*}\left(0 \leq t^{*} \leq t^{\mathrm{m}}\right)$ using cubic fit (see Appendix B for more details). Calculate $\boldsymbol{\Delta} \mathbf{P}=t^{*} \mathbf{S}$. Update power injections $\mathbf{P}^{\mathrm{s}}=\mathbf{P}^{\mathrm{s}}+\boldsymbol{\Delta} \mathbf{P}$. Vector $\boldsymbol{\Delta} \mathbf{P}$ can include generation rescheduling and/or load shedding, depending on the current strategy $s p$;

10 Calculate the objective function value, $f_{1}$. If $f_{1} \leq$ $\epsilon_{1}$ or $\left[\left(f_{1}-f_{0}\right) / f_{1}\right] \leq \epsilon_{2}$ go to 11. Otherwise, increment inner iteration counter $i c=i c+1$ and go to step 4;

11 Perform load flow calculations for the new generation and load schedule. Set counters $o c=o c+1$ and $s p=s p+1$. Go to step $\mathbf{2}$;

12 Compute the power injection at the slack bus. In case it is outside bounds, curtail load at the slack bus itself and/or at buses which are fed from the slack bus. The amount of load shedding is equal to the limit violation. Else stop.

\section{HEURISTICS}

The following heuristics were added to increase efficency and to take into account some practical aspects related to the overload elimination problem.

- Step 2: in many cases it is possible that $t^{*}$ be determined so as to zero the objective function, that is, the control actions are expected to successfully eliminate the overloads. However, after performing load flow calculations one can find out that the overloads have not been eliminated, but only alleviated. This occurs due to the utilization of the real part $(P-\theta)$ of the load flow equations in the optimization process. In case this situation occurs, set $s p=s p-1$. Decreasing $s p$ implies in performing another iteration by using the same control strategy as in the previous one, and the error is corrected;

- Step 2: if $o c>1$ and new overloaded branches are detected (those that appeared only after control actions have been taken) such that the value of the objective function increases, steps 3-10 are performed considering only the new overloaded branches for computing the objective function. After the new overloads had been eliminated, all controls used at this specific step are flagged. These controls cannot be changed in further iterations so that the same new overloads appear;

- Step 9: in case the derivative of the objective function evaluated at $t=t^{\mathrm{m}}$ is less or equal to zero, set $t^{*}=t^{\mathrm{m}}$. Its negative sign indicates that the objective function is still decreasing when some control limit has been reached. In this case some computation savings are achieved;

- Step 11: if the load flow calculations do not converge, set the controls back to their previous values in the reverse order they appeared until convergence is obtained.

\section{GENERAL COMMENTS}

It follows general comments regarding the implementation of the proposed method.

- The elements of vector $\boldsymbol{\lambda}$ in steps $\mathbf{4}$ and $\mathbf{5}$ are efficiently computed by using sparse vector techniques. Vector $\partial f / \partial \boldsymbol{\theta}$ is sparse, so fast forward substitution can be performed. If a small number of elements of $\boldsymbol{\lambda}$ is required, fast back substitution can be performed;

- The threshold of step $\mathbf{5}$ is defined as a percentage of either the largest lagrangean multiplier related to the terminal buses of the overloaded branches or the lagrangean multiplier related to the first generator found;

- Tolerances $\epsilon_{1}$ and $\epsilon_{2}$ are arbitrarily chosen. In this paper $\epsilon_{1}=10^{-6}$ and $\epsilon_{2}=10^{-2}$ were used with good final results.

- The overload elimination problem was formulated using the well known idea of the strong real power/ 
phase angle coupling (Sec. 2). This feature of the problem allows the use of first order optimization methods, such as the steepest descent method used in this paper. The usually undesirable characteristics of first order methods, such as slow convergence, are not critical in this case. Nevertheless, the idea of adaptive local optimization can be used in conjunction with any optimization method, such as the second order Newton method (Sun et alii, 1984) and the interior point method (Ponnambalam et alii, 1991).

\section{RESULTS OF SIMULATIONS}

The simulation results shown in this paper are restricted to two power systems, namely the IEEE 300 bus, 411 branch system (http://www.ee.washington.edu/research) and a 904 bus, 1283 branch system which corresponds to a reduced version of the Southwestern USA system.

\subsection{ALO versus LO}

Table 1 shows a comparison between the concepts of ALO and LO using the 904 bus system. Branch 849851 is overloaded (original flow is $523.07 \mathrm{MW}$, limit set to $520.00 \mathrm{MW}$ ).

Table 1: ALO versus LO

\begin{tabular}{l|l|l|l|l}
\hline & \multicolumn{2}{|c|}{ ALO } & \multicolumn{2}{c}{ LO } \\
\hline \hline$o c, i$ & 1,1 & 2,1 & 1,1 & 2,1 \\
\hline$s p$ & 1 & 1 & 1 & 2 \\
\hline control bus & $17,18\left(^{*}\right)$ & 17, & - & $849\left(^{*}\right)$ \\
& & $18\left(^{*}\right)$ & & \\
\hline tier & 3 & 3 & - & 0 \\
\hline control ac- & $-23.13(\mathrm{r}) ;$ & $-0.85(\mathrm{r}) ;$ & - & $40.18(\mathrm{l})$ \\
tion & $-23.13(\mathrm{r})$ & $-0.85(\mathrm{r})$ & & \\
\hline power flow & 520.11 & 520.00 & 523.07 & 519.76 \\
\hline \hline
\end{tabular}

(*) bus that limited $t^{\mathrm{m}} ;(\mathrm{r})$ rescheduling;

(l) load shedding

In the case of LO, the work region was defined with 2 tiers, as suggested in (Shandilya et alii, 1993). In this case, no rescheduling was possible since there were no generators within the work area. The next step is to curtail load at the terminal bus of the overloaded branch. By using ALO, generators at the third tier were found and were able to eliminate the overload without load shedding. Generators 17 and 18 have the same sensitivities, resulting in identical rescheduling.

\subsection{Multiple overloads}

Table 2 shows simulation results for a double overload at branches 851-849 (original flow is 523.07 MW, limit set to $520.00 \mathrm{MW}$ ) and $849-890$ (original flow is 70.36 MW, limit set to $67.00 \mathrm{MW}$ ) of the 904 bus system.

Table 2: Two overloads

\begin{tabular}{l|l|l|l|l}
\hline$o c, i$ & 1,1 & 1,2 & 1,3 & 2,1 \\
\hline$s p$ & 1 & 1 & 1 & 1 \\
\hline control & $2\left(^{*}\right), \quad 17$, & $2\left(^{*}\right), 17$, & $2\left(^{*}\right), 17$, & $2\left(^{*}\right), 17$, \\
bus & 18 & 18 & 18 & 18 \\
\hline control & $-16.84(\mathrm{r})$, & $+10.38(\mathrm{r})$, & $-0.75(\mathrm{r})$, & $-0.78(\mathrm{r})$, \\
action & $-16.18(\mathrm{r})$, & $-4.72(\mathrm{r})$, & $-0.81(\mathrm{r})$, & $-0.87(\mathrm{r})$, \\
& $-16.18(\mathrm{r})$ & $-4.72(\mathrm{r})$ & $-0.81(\mathrm{r})$ & $-0.87(\mathrm{r})$ \\
\hline linearized & 519.60, & 520.14, & 519.96, & 520.00, \\
flows & 67.61 & 67.18 & 67.05 & 67.00 \\
\hline actual & - & - & 520.19, & 519.96, \\
flows & & & 67.14 & 67.06 \\
\hline \hline
\end{tabular}

After three inner iterations, control actions are taken so as to eliminate the overloads. However, by solving the load flow equations, it is found that the overloads are still present (though smaller than the original ones). Control actions are defined within an inner iteration by linearized calculations. There may be a difference between the expected flows and the ones calculated by solving the load flow equations. Another external iteration is necessary to finally eliminate the violation. In fact, it can be seen that there is a small overload left in branch 849-890. However, the stopping criteria is met, that is, the objective function is less or equal to $\epsilon_{1}$.

\subsection{New overloads}

Table 3 shows the results of a simulation of an overload at branch 849-851 (original flow is 523.07 MW, limit set to $520.00 \mathrm{MW}$ ) of the 904 bus system. Branch 851-824 (original flow is $78.26 \mathrm{MW}$, limit set to $80 \mathrm{MW}$ ) has also been monitored since it becomes overloaded after some control actions.

The first control action results in a new overload at branch 851-824 such that the value of the objective functions increases. The new overload is eliminated at iteration 2. Further load shedding is necessary for a complete elimination of the overloads. Note that branch 849-851 ends up with a small overload, however, within acceptable limits, defined by tolerance $\epsilon_{1}$. 
Table 3: New overload

\begin{tabular}{l|l|l|l|l|l}
\hline$o c$ & $s p$ & $\begin{array}{l}\text { control } \\
\text { buses }\end{array}$ & $\begin{array}{l}\text { overload } \\
\text { considered }\end{array}$ & $\begin{array}{l}\text { control } \\
\text { action }\end{array}$ & $\begin{array}{l}\text { actual } \\
\text { flows }\end{array}$ \\
\hline \hline 1 & 1 & $17 ;$ & $849-851$ & - \\
& & $18\left(^{*}\right)$ & & $\begin{array}{l}23.13(\mathrm{r}) ; \\
-23.13(\mathrm{r})\end{array}$ & $\begin{array}{l}520.11 ; \\
84.45\end{array}$ \\
\hline 2 & 1 & $2\left(^{*}\right) ; 17 ;$ & $851-824$ & $\begin{array}{l}-9.01(\mathrm{r}) ; \\
14.51(\mathrm{r}) ;\end{array}$ & $\begin{array}{l}521.84 ; \\
79.84\end{array}$ \\
& & 18 & & $14.51(\mathrm{r})$ & \\
\hline 3 & 1 & - & $849-851$ & - & $521.84 ;$ \\
& & & & & 79.84 \\
\hline 4 & 2 & $849\left(^{*}\right)$ & $849-851$ & $24.10(\mathrm{l})$ & $519.85 ;$ \\
& & & & & 81.41 \\
\hline 5 & 2 & $824\left(^{*}\right)$ & $851-824$ & $5.13(\mathrm{l})$ & $520.43 ;$ \\
& & & & & 79.96 \\
\hline 6 & 2 & $849\left(^{*}\right)$ & $849-851$ & $5.64(\mathrm{l})$ & $519.97 ;$ \\
& & & & & 80.32 \\
\hline 7 & 2 & $824\left(^{*}\right)$ & $851-824$ & $1.18(\mathrm{l})$ & $520.10 ;$ \\
& & & & & 79.99 \\
\hline \hline
\end{tabular}

\subsection{Emergency control measures}

Table 4 shows the results provided by the proposed method for the IEEE 300 bus system. Branch 2-8 (original flow is $365.11 \mathrm{MW}$, limit set to $350 \mathrm{MW}$ ) is overloaded.

Table 4: Extreme control mesures

\begin{tabular}{l|l|l|l|l}
\hline$o c$ & 1 & 2 & 3 & 4 \\
\hline$s p$ & 1 & 2 & 3 & 4 \\
\hline $\begin{array}{l}\text { control } \\
\text { buses }\end{array}$ & $7002\left(^{*}\right)$ & $8\left(^{*}\right)$ & - & $7003,9,13$, \\
& & & & $15\left(^{*}\right)$ \\
\hline control & - & $10.00(\mathrm{l})$ & - & $-15.83(\mathrm{r})$, \\
action & $23.00(\mathrm{r})$ & & & $4.92(1)$, \\
& & & & $5.62(\mathrm{l})$, \\
& & & & $15.69(\mathrm{l})$ \\
\hline $\begin{array}{l}\text { actual } \\
\text { flows }\end{array}$ & 357.74 & 353.37 & 353.37 & 349.86 \\
\hline \hline
\end{tabular}

In the first iteration generator 7002 is rescheduled down to its lower limit. In iteration 2, receiving end bus number 8 undergoes load shedding. In iteration 3 , buses fed from bus 8 (first tier) are checked and no load shedding is possible, however, the overload is still in effect. Iteration 4 constitutes an emergency control measure, that is, generation rescheduling and load shedding is allowed also for buses belonging up to the third tier, and the overload is eliminated through rescheduling of the generator at bus 7003 and load shedding at buses 9,13 and 15.

\subsection{Performance aspects}

Some performance aspects will be discussed based on the same simulation shown in Section 6.4. In the first outer iteration $(o c=1)$ generator rescheduling was allowed only. The search for generators started from the terminal buses of the overloaded branch and ended up in the third tier, as shown in Figure 1. Generators 8, $7002,7003,10,7011,7001$ and 20 were found. All of them, except generator 7002 either had small sensitivities or could not be rescheduled due to their particular generation limits. Therefore, the work region consisted of generator bus 7002 and terminal buses 2 and 8 . In order to find out the work region (and also vector $\boldsymbol{\lambda}$ ) one fast forward (for the factorization path corresponding to buses 2 and 8 ) and six fast back (for the factorization paths corresponding to each one of the generators) substitutions were performed.

In all further iterations only one fast forward and one fast back substitutions had to be performed for obtaining $\boldsymbol{\lambda}$. Of course, the factorization paths were different in each case depending on the buses that were considered (2, 5 and 11 buses in iterations 2,3 and 4, respectively).

In this particular simulation, a region containing 20 buses (less than $10 \%$ of the total number of buses) was sufficient for solving the problem. Even though the size of the work region and the number of buses that have to be considered depend on the system and the overload, similar results have been obtained for all systems tested. Actually, the larger the system, the smaller the work region in proportional terms. Therefore, the computational burden can be significantly reduced by using the concept of adaptive local optimization.

\section{CONCLUSIONS}

In this paper a new nonlinear programming based method for eliminating branch overloads was proposed. Several heuristics were added to the basic projected steepest descent algorithm in order to take into account some practical aspects of power systems operation and to increase its computational efficiency. Regarding the later, the ideas of adaptive local optimization and sparse vector techniques were also used. The method proved to be robust and efficient, being able to deal with complex overload situations, providing fast and reliable solutions.

\section{ACKNOWLEDGEMENTS}

The authors thank the financial support provided by FAPESP (Fundação de Amparo à Pesquisa do Estado de São Paulo), Brazil. 


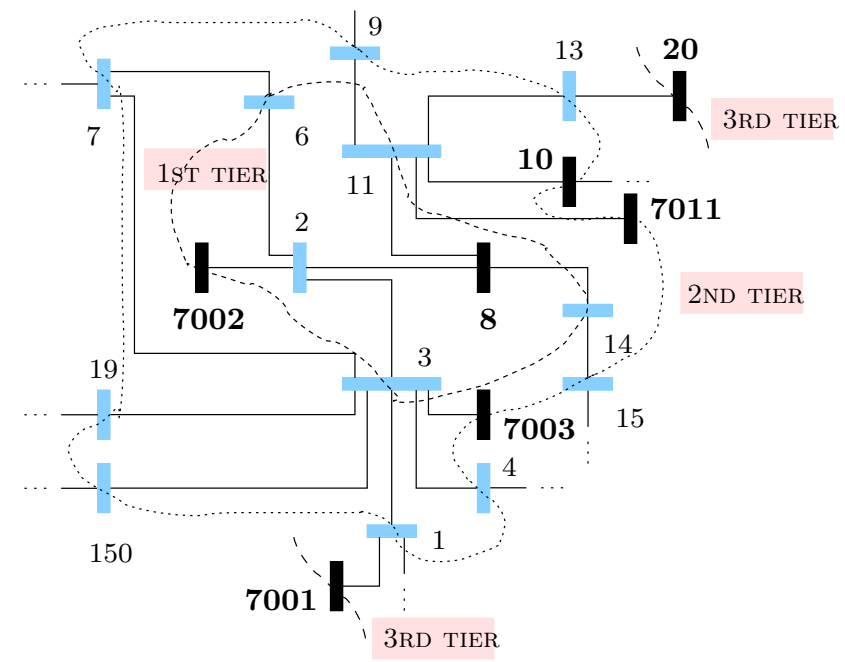

Figure 1: Work region

\section{REFERENCES}

Bazaraa, M. and Shetty, C.M. (1993) Nonlinear programming - theory and algorithms, John Willey and Sons.

Castro, C.A. and Bose, A. (1993) Correctability in online contingency analisys. IEEE Transactions on Power Systems 8(3): 807-813.

Castro, C.A. and Bose, A. (1994) Correctability of voltage violations in on-line contingency analisys. IEEE Transactions on Power Systems 9(3): 1651-1657.

Luenberger D. (1984) Linear and nonlinear programming, Addison-Wesley.

Medicherla, T.K.P., Billinton, R. and Sachadev, M.S. (1979) Generation rescheduling and load shedding to alleviate line overloads. IEEE Transactions on Power Apparatus and Systems 98(6): 1876-1884.

Ponnambalam K., Quintana V.H. and Vannelli A. (1991) A fast algorithm for power system optimization problems using an interior point method. Proc. of the Power Industry Computer Applications Conference, 393-400.

Shandilya, A., Gupta, H. and Sharma, J. (1993) Method for generation rescheduling and load shedding to alleviate line overloads using local optimization. IEE Proceedings - C 140(5): 337-342.

Stott, B. and Alsaç, O. (1974) Fast decoupled load flow. IEEE Transactions on Power Apparatus and Systems 93(3): 859-869.
Sun D., Ashley B., Brewer B., Hughes A. and Tinney W.F. (1984) Optimal power flow by Newton approach. IEEE Transactions on Power Apparatus and Systems 103(10): 1248-1259.

Tinney, W.F., Brandwajn, V. and Chan, S.M. (1985) Sparse vector methods. IEEE Transactions on Power Apparatus and Systems 104(2): 295-301.

Wood, A.J. and Wollenberg, B.F. (1984) Power generation, operation and control, John Willey and Sons.

\section{APPENDIX A OPTIMALITY CONDI- TIONS}

The Lagrangean function corresponding to problem (5)(7) is:

$$
\mathcal{L}\left(\boldsymbol{\theta}, \mathbf{P}^{\mathrm{s}}, \boldsymbol{\lambda}\right)=f(\boldsymbol{\theta})+\boldsymbol{\lambda}^{T} \cdot \mathbf{g}\left(\boldsymbol{\theta}, \mathbf{P}^{\mathrm{s}}\right)
$$

Applying the optimality conditions one gets:

$$
\begin{array}{cc}
\frac{\partial \mathcal{L}}{\partial \boldsymbol{\theta}} & =\frac{\partial f}{\partial \boldsymbol{\theta}}+\left(\frac{\partial \mathbf{g}}{\partial \boldsymbol{\theta}}\right)^{T} \cdot \boldsymbol{\lambda}=0 \\
\frac{\partial \mathcal{L}}{\partial \boldsymbol{\lambda}}= & \mathbf{g}\left(\boldsymbol{\theta}, \mathbf{P}^{\mathrm{s}}\right)=0 \\
\frac{\partial \mathcal{L}}{\partial \mathbf{P}^{\mathrm{s}}}=\left(\frac{\partial \mathbf{g}}{\partial \mathbf{P}^{\mathrm{s}}}\right)^{T} \cdot \boldsymbol{\lambda} & =0
\end{array}
$$

From (9):

$$
\boldsymbol{\lambda}=-\left[\left(\frac{\partial \mathbf{g}}{\partial \boldsymbol{\theta}}\right)^{T}\right]^{-1} \cdot \frac{\partial f}{\partial \boldsymbol{\theta}}
$$

From (11) and (12):

$$
\frac{\partial \mathcal{L}}{\partial \mathbf{P}^{\mathrm{s}}}=-\left(\frac{\partial \mathbf{g}}{\partial \mathbf{P}^{\mathrm{s}}}\right)^{T} \cdot\left[\left(\frac{\partial \mathbf{g}}{\partial \boldsymbol{\theta}}\right)^{T}\right]^{-1} \cdot \frac{\partial f}{\partial \boldsymbol{\theta}}
$$

Matrix $\partial \mathbf{g} / \partial \mathbf{P}^{\mathrm{s}}$ is an identity matrix. Since only the real power part of the load flow equations are used, matrix $[\partial \mathbf{g} / \partial \boldsymbol{\theta}]^{T}$ is replaced by the fast decoupled load flow matrix $\left(-\mathbf{B}^{\prime}\right)$ (Stott and Alsaç, 1974). $\mathbf{B}^{\prime}$ is a symmetrical matrix with constant entries, and it is factorized only once. This feature results in important computational savings. Finally, Eq. (13) can be written as:

$$
\boldsymbol{\Delta} \mathcal{L}_{P}=\frac{\partial \mathcal{L}}{\partial \mathbf{P}^{\mathrm{s}}}=\mathbf{B}^{\prime-1} \cdot \frac{\partial f}{\partial \boldsymbol{\theta}}
$$

Vector $\boldsymbol{\Delta} \mathcal{L}_{P}$ provides an indication of how far the problem is from the optimal conditions. 


\section{APPENDIX B CUBIC FIT}

Suppose that two points $x_{0}$ and $x_{\mathrm{m}}$ are known. Suppose also that it is possible to calculate the values of a function $f(x)$ at each of these points $\left(f\left(x_{0}\right)\right.$ and $\left.f\left(x_{\mathrm{m}}\right)\right)$, as well as the values of their respective derivatives $\left(f^{\prime}\left(x_{0}\right)\right.$ and $\left.f^{\prime}\left(x_{\mathrm{m}}\right)\right)$. The main idea is to fit a cubic equation to these points (Luenberger, 1984). Then, a third point $x_{*}$ can be found such that $f\left(x_{*}\right)$ will be the minimum of $f(x)$. Point $x_{*}$ is obtained by:

$$
x_{*}=x_{\mathrm{m}}-\left(x_{\mathrm{m}}-x_{0}\right)\left[\frac{f^{\prime}\left(x_{\mathrm{m}}\right)+u_{2}-u_{1}}{f^{\prime}\left(x_{\mathrm{m}}\right)-f^{\prime}\left(x_{0}\right)+2 u_{2}}\right]
$$

where:

$$
\begin{aligned}
& u_{1}=f^{\prime}\left(x_{0}\right)+f^{\prime}\left(x_{\mathrm{m}}\right)-3\left[\frac{f\left(x_{0}\right)-f\left(x_{\mathrm{m}}\right)}{x_{0}-x_{\mathrm{m}}}\right] \\
& u_{2}=\left[u_{1}^{2}-f^{\prime}\left(x_{0}\right) f^{\prime}\left(x_{\mathrm{m}}\right)\right]^{1 / 2}
\end{aligned}
$$

There are two considerations about this method: (a) it is exact for quadratic functions (quadratic convergence); (b) it has been formulated for a function of one variable.

The objective function $f$ in this paper depends on the bus phase angles vector $\boldsymbol{\theta}$. More specifically, it depends on two bus phase angles per overload $\left(\theta_{k}\right.$ and $\theta_{m}$ for an overloaded branch connecting buses $k$ and $m$ ). In order to use the cubit fit Eqs. (15)-(17) for the determination of $t^{*}$ in step 9 of the proposed algorithm, it is necessary to alter the objective function in such a way that it becomes a single variable function. Particularly, $f$ must be formulated as a function of the step size $t$. For the sake of simplicity, consider an overload at branch $\ell$, which connects buses $k$ and $m$. The objective function is:

$$
f(\boldsymbol{\theta})=\left(P_{\ell}^{0}(\boldsymbol{\theta})-P_{\ell}^{\lim }\right)^{2}
$$

The MW power flow limit $P_{\ell}^{\text {lim }}$ is constant. The MW power flow $P_{\ell}^{0}(\boldsymbol{\theta})$ through branch $\ell$ can be written in its linearized form as:

$$
P_{\ell}^{0}(\boldsymbol{\theta})=\frac{\theta_{k}-\theta_{m}}{x_{k m}}
$$

where $x_{k m}$ is the branch series reactance. After some control action is taken, the state of the system will change and the new MW power flow through branch $\ell$ will be:

$$
\begin{aligned}
P_{\ell}^{1}(\boldsymbol{\theta}) & =\frac{\left(\theta_{k}+\Delta \theta_{k}\right)-\left(\theta_{m}+\Delta \theta_{m}\right)}{x_{k m}} \\
& =P_{\ell}^{0}(\boldsymbol{\theta})+\frac{\Delta \theta_{k}-\Delta \theta_{m}}{x_{k m}} \\
& =P_{\ell}^{0}(\boldsymbol{\theta})+\frac{1}{x_{k m}} \cdot \mathbf{e}_{k m}^{T} \cdot \boldsymbol{\Delta} \boldsymbol{\theta}
\end{aligned}
$$

where $\mathbf{e}_{k m}^{T}$ is a column vector with 1 and -1 at positions $k$ and $m$ respectively and zeros elsewhere. Vector $\boldsymbol{\Delta} \boldsymbol{\theta}$ is computed by (Stott and Alsaç, 1974):

$$
\Delta \theta=\mathbf{B}^{\prime-1} \cdot \Delta \mathbf{P}
$$

and $\mathbf{\Delta} \mathbf{P}$ is obtained by (step $\mathbf{9}$ of the proposed algorithm):

$$
\Delta \mathbf{P}=t \cdot \mathbf{S}=-t \cdot \boldsymbol{\lambda}
$$

Thus, the new objective function can be written as a function of step size $t$ only, by substituting eqs. (20), (21) and (22) into (18):

$$
\begin{aligned}
f(t) & =\left(P_{\ell}^{1}(\boldsymbol{\theta})-P_{\ell}^{\lim }\right)^{2} \\
& =\left(P_{\ell}^{0}-P_{\ell}^{\lim }+\frac{1}{x_{k m}} \mathbf{e}_{k m}^{T} \mathbf{B}^{\prime-1} \mathbf{S} t\right)^{2}
\end{aligned}
$$

Let $\boldsymbol{\theta}_{0}$ be the vector of bus phase angles corresponding to a certain operating point for which overloads do exist. In this case the objective function is $f\left(\boldsymbol{\theta}_{0}\right)$ or $f(0)$, depending on whether Eq. (18) or Eq. (23) is used, respectively. In step $\mathbf{6}$ of the proposed algorithm vector $\mathbf{S}$ is computed, indicating the direction of control changes along which the objective function will decrease. In step 8 the maximum step size $t^{\mathrm{m}}$ is computed based on sensitivity factors and generator availabilities. Let $\boldsymbol{\theta}_{\mathrm{m}}$ be the vector of bus phase angles corresponding to a maximum change in controls. Let also $f\left(\boldsymbol{\theta}_{\mathrm{m}}\right)$ (or $\left.f\left(t^{\mathrm{m}}\right)\right)$ be the respective value of the objective function.

In step 9 the optimum step size $t^{*}\left(0 \leq t^{*} \leq t^{\mathrm{m}}\right)$ must be obtained. $t^{*}$ must result in a certain control action such that $f\left(\boldsymbol{\theta}_{*}\right)$ (or $f\left(t^{*}\right)$ ) be the minimum of $f(\boldsymbol{\theta})$, where $\boldsymbol{\theta}_{*}$ is the vector of bus phase angles after a control action based on $t^{*}$ is taken into effect. In this case, Eqs. (15)(17) become:

$$
\begin{aligned}
& u_{1}=f^{\prime}\left(t^{0}\right)+f^{\prime}\left(t^{\mathrm{m}}\right)-3\left[\frac{f\left(t^{0}\right)-f\left(t^{\mathrm{m}}\right)}{t^{0}-t^{\mathrm{m}}}\right] \\
& u_{2}=\left[u_{1}^{2}-f^{\prime}\left(t^{0}\right) f^{\prime}\left(t^{\mathrm{m}}\right)\right]^{1 / 2} \\
& t^{*}=t^{\mathrm{m}}-\left(t^{\mathrm{m}}-t^{0}\right)\left[\frac{f^{\prime}\left(t^{\mathrm{m}}\right)+u_{2}-u_{1}}{f^{\prime}\left(t^{\mathrm{m}}\right)-f^{\prime}\left(t^{0}\right)+2 u_{2}}\right]
\end{aligned}
$$

